Forum 2021 · 36:183

https://doi.org/10.1007/s12312-021-00941-0

(c) Springer Medizin Verlag GmbH, ein Teil von Springer Nature 2021

Liebe Kolleginnen, liebe Kollegen,

die Antwort auf die Frage, wie alt man sich fühlt, dürfte bei den meisten Menschen z. B. in Abhängigkeit von Tagesform, Arbeitspensum oder dem Alter der Studenten, die immer jünger zu werden scheinen, variieren. Die Frage nach dem subjektiven Altersgefühl ist keineswegs eine banale. Warum sind manche Menschen auch im höheren Alter widerstandsfähiger als andere? Welche Rolle spielen die Genetik und die Persönlichkeit, das soziale Umfeld, Lebensstilentscheidungen und letztlich die Geisteshaltung für die Tatsache, wie man altert und ob man das Alter als eine Bürde betrachtet oder genießen kann?

Angesichts der steigenden Lebenserwartung und der Zunahme von chronischen Krankheiten wie Krebs ist die gute medizinische und pflegerische Versorgung von alten Menschen eine hochrelevante Aufgabe. Ältere Patienten mit einer Krebserkrankung sind eine heterogene Gruppe mit unterschiedlichen altersspezifischen Eigenschaften. Merkmale wie Allgemeinzustand, körperliche Fitness versus Gebrechlichkeit, geistige Flexibilität, veränderte Organfunktionen oder die Selbstständigkeit im Alltag haben Einfluss auf die Therapiefähigkeit.

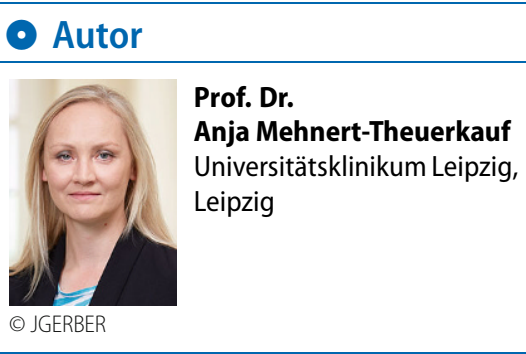

\title{
Anja Mehnert-Theuerkauf
}

Abt. für Medizinische Psychologie und Medizinische Soziologie, Universitätsklinikum Leipzig, Leipzig, Deutschland

\section{Wie alt wären Sie, würden Sie nicht wissen, wie alt Sie sind?}

Zwei ältere Patienten gleichen Alters erhalten zur Behandlung einer malignen Tumorerkrankung in einem Krebszentrum mehr oder weniger die gleiche medizinische Versorgung. Dabei ist die Gebrechlichkeit eines Patienten ein wichtiger und frühzeitiger Indikator dafür, wie die Therapie anschlagen und sich auf die Lebensqualität auswirken wird. Für manchmal schwierige Therapieentscheidungen bei älteren Menschen spielt die Abwägung zwischen der Toxizität der Behandlungsoptionen und den Lebensqualitätseinschränkungen eine wichtige Rolle, v. a. mit Blick auf den Erhalt der Funktionsfähigkeit und der Selbstständigkeit im Alltag.

Gebrechlichkeit stellt eine große Herausforderung für die gesundheitliche Versorgung älterer Menschen dar. Zum einen ist Gebrechlichkeit stark mit dem Altern verbunden und die Zahl der älteren Patienten nimmt stetig zu. Zum anderen bedeutet ein höheres Lebensalter nicht zwangsläufig eine Zunahme an Gebrechlichkeit. Getreu dem nicht ganz ernst gemeinten Motto einer internationalen Tageszeitung „70 ist das neue 30" gibt es dennoch eine steigende Zahl rüstiger Älterer.

Die frühzeitige Gesundheitsförderung spielt nicht nur bei der Prävention von Krebserkrankungen eine wichtige Rolle, sondern auch bei der Verringerung des Schweregrads der Gebrechlichkeit. Lebensstilfaktoren wie Bewegung und regelmäßige körperliche Aktivität, gute Ernährung und Resilienz, d.h. die psychische Widerstandsfähigkeit, scheinen von besonderer Bedeutung zu sein. Der Aufbau und Erhalt von sinnstiftenden Beziehungen $\mathrm{zu}$ anderen Menschen sowie die Kultivierung einer positiven, lebensbejahenden inneren Haltung haben Einfluss darauf, ob ältere Menschen aktiv und vital bleiben. Kurz: Die Förderung eines stabilen Gesundheitszustands durch gesündere Lebensgewohnheiten und Lebenszufriedenheit könnte die Anfälligkeit für Gebrechlichkeit und Multimorbidität verringern. Altern mag unvermeidlich sein, aber Gebrechlichkeit ist es nicht.

Ich wünsche Ihnen viel Freude und neue Erkenntnisse beim Lesen der interessanten und anregenden Beiträge dieses Hefts zum Thema geriatrische Onkologie.

Ihre

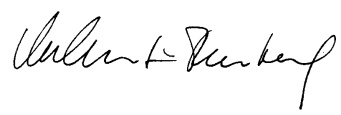

Anja Mehnert-Theuerkauf

\section{Korrespondenzadresse}

Prof. Dr. Anja Mehnert-Theuerkauf Abt. für Medizinische Psychologie und Medizinische Soziologie, Universitätsklinikum Leipzig

Philipp-Rosenthal-Straße 55, 04103 Leipzig, Deutschland

anja.mehnert@medizin.uni-leipzig.de

Interessenkonflikt. A. Mehnert-Theuerkauf gibt an, dass kein Interessenkonflikt besteht. 